\title{
Innate Immunity of the Human Newborn Is Polarized Toward a High Ratio of IL-6/TNF- $\alpha$ Production In Vitro and In Vivo
}

\author{
DONATELLA F. ANGELONE, MICHAEL R. WESSELS, MELISSA COUGHLIN, EUGENIE E. SUTER, PIERO VALENTINI, \\ LESLIE A. KALISH, AND OFER LEVY \\ Catholic University of the Sacred Heart [D.A., P.V.], 00168 Rome, Italy; Children's Hospital Boston [M.W., M.C., E.E.S., LAK.], \\ Harvard Medical School [M.W., LAK., O.L.], Boston, Massachusetts, 02115
}

\begin{abstract}
Human newborns are susceptible to microbial infection related to incompletely defined aspects of the neonatal immune system. To characterize neonatal innate immunity, we studied production of two early response cytokines in response to Toll-like receptor (TLR)-activating microbial stimuli in vitro: the proinflammatory cytokine tumor necrosis factor (TNF)- $\alpha$ and IL-6, a multifunctional cytokine with antiinflammatory and Th2-polarizing properties. Neonatal cord blood responses to multiple TLR agonists, including poly dI:dC (TLR3), lipopolysaccharide (LPS) (TLR4), flagellin (TLR5), and CpG DNA (TLR9), are characterized by a higher IL-6/TNF- $\alpha$ ratio than in adult peripheral blood. Robust LPS-induced IL-6 production is due to both neonatal cellular (monocyte-) and humoral (serum-) factors. Remarkably, serum collected from newborns during the first week of life demonstrates higher IL-6/TNF- $\alpha$ ratios than does cord blood, associated with elevations of the IL-6-inducible acute phase reactants CRP and LPS-binding protein in the first days of life. A high ratio of stimulus-induced IL-6/TNF- $\alpha$ production is likely to profoundly modulate both innate and adaptive immune responses in the human newborn. (Pediatr Res 60: 205-209, 2006)
\end{abstract}

$\mathrm{I}^{\mathrm{n}}$ ncreased susceptibility of human newborns to microbial infection has been ascribed in part to impaired Th1-type adaptive immune responses $(1,2)$. In addition, several studies have demonstrated impaired stimulus-induced early cytokine responses, including endotoxin-induced production of the proinflammatory and Th1-polarizing cytokine TNF- $\alpha$ from neonatal cells (3-5), whereas others have suggested that neonatal production of IL-6, a cytokine with antiinflammatory (6) and Th2-polarizing properties $(7)$, may be enhanced $(8,9)$. However, a direct comparison of neonatal production of these early cytokines to well-defined microbial products and the demonstration of such a pattern in vivo has been lacking.

The discovery of the TLR family of proteins (10) offers an important opportunity to gain new insights into neonatal innate immune responses. Humans express 10 TLR that serve to recognize an array of microbial products and trigger intracellular signaling cascades culminating in nuclear translocation of nuclear factor (NF)- $\kappa \mathrm{B}$ and consequent expression of genes

Received December 16, 2005; accepted March 28, 2006.

Correspondence: Ofer Levy, M.D., Ph.D., Children's Hospital Boston, Division of Infectious Diseases, 300 Longwood Avenue, Boston, MA 02115; e-mail: ofer.levy@childrens.harvard.edu

This study was supported by National Institutes of Health grant number KO8 AI50583-01 to OL and a Scholarship for Pediatric Residency (Italy) to DA.

DOI: 10.1203/01.pdr.0000228319.10481.ea mediating the inflammatory response. We have previously shown that, despite normal basal expression of TLR and associated signaling intermediates, neonatal responses to a range of pure TLR agonists are characterized by impaired production of TNF- $\alpha$ (11). Our study raised the question of whether TLR-induced production of cytokines with distinct function was also impaired in neonates and whether the patterns of cytokine production measured in vitro were also evident in vivo.

In the present study, we therefore examined neonatal and adult production of TNF- $\alpha$ in comparison to IL-6, a multifunctional cytokine that induces acute phase production of CRP and LBP (12) and that also has Th2-polarizing (13) and antiinflammatory properties $(6,7)$. We report here that the innate immune responses of neonatal monocytes to microbial TLR agonists are biased toward a high IL-6/TNF- $\alpha$ ratio in vitro, which is due to distinct neonatal cellular (monocyte) and humoral (serum) factors. Moreover, we find that such a pattern of high IL-6 but low TNF- $\alpha$ production is evident in the early postnatal phase in vivo, revealing a physiologically relevant pattern that is likely to profoundly modulate innate and adaptive responses.

\section{METHODS}

Blood. Peripheral blood from healthy adult volunteers ( $n=23$; mean age, $27 \mathrm{y})$ and newborn cord blood ( $n=23$, mean GA, $37 \mathrm{wk}$; range, 36-40 wk) collected immediately after cesarean section delivery of the placenta were obtained in Boston, MA. Births at which antibiotics were administered during labor/delivery, and births to HIV-positive mothers were excluded. Human experimentation guidelines of the U.S. Department of Health and Human Services were observed, following Institutional Review Board-approved protocols. Blood was anticoagulated with $109 \mathrm{mM}$ sodium citrate. For serum preparation, blood was collected in sterile tubes without additives, allowed to clot for $30 \mathrm{~min}$, then centrifuged $(2000 \mathrm{rpm}, 20 \mathrm{~min})$ before collection and storage at $-80^{\circ} \mathrm{C}$. For experiments determining the IL- 6 stimulatory activity of heat-treated serum, fresh neonatal or adult serum was preincubated at $56^{\circ} \mathrm{C}$ for $30 \mathrm{~min}$ (or, for comparison, at $4^{\circ} \mathrm{C}$ ) before addition to cells. All blood collection tubes were from BD Biosciences (Franklin Lakes, NJ).

Monocyte purification. Blood was collected into sterile, pyrogen-free sodium heparin (10 U/mL; American Pharmaceutical Partners, Schaumberg, IL), peripheral blood mononuclear cells (PBMC) were prepared using Ficoll-

Abbreviations: CRP, C-reactive protein; GA, gestational age; LBP, LPSbinding protein; LPS, lipopolysaccharide; TLR, Toll-like receptor; TNF- $\alpha$, tumor necrosis factor-alpha 
Hypaque gradients (Sigma Chemical Co., St. Louis, MO) and monocytes isolated by positive selection using anti-CD14-conjugated magnetic microbeads (Millenyi Biotec, Sunnyvale, CA) as previously described (11). Monocytes purified by this positive selection protocol exhibit normal forward- and side-scatter properties by flow cytometry, stain normally for CD14, and have undetectable basal production of TNF- $\alpha$ (11), suggesting that the isolation procedure does not lead to cellular activation.

TLR agonists. Double-stranded RNA poly (I:C) (TLR3) was tested at 1, 10 , and $100 \mu \mathrm{g} / \mathrm{mL}$ (InvivoGen, San Diego, CA), CpG oligodeoxynucleotide 2216 (TLR9) at 10 and $20 \mu \mathrm{M}$ (InvivoGen), and ultra-pure Re595 LPS (TLR4) from Salmonella minnesota at $0.1,1$, and $10 \mathrm{ng} / \mathrm{mL}$ (List Biologicals, Campbell, CA). The specificity of LPS to TLR4 was determined by assessing LPS-induced activation of TLR4- but not TLR2-transfected human embryonic kidney cells as previously described (11). Recombinant flagellin (TLR5), a generous gift of Dr. Steven Mizel (Wake Forest University, Winston-Salem, NC), was expressed and purified as previously described (14) and tested at 10 and $100 \mathrm{nM}$.

In vitro stimulation of leukocytes. Studies of in vitro stimulation used cord blood (or cord blood-derived leukocytes) from neonates born in Boston. Whole blood or purified monocytes cultured in fresh autologous or heterologous serum $\left(10^{7}\right.$ cells $\left./ \mathrm{mL}\right)$ were stimulated with agonists for $1,3,5,8$, or $24 \mathrm{~h}$ at $37^{\circ} \mathrm{C}$ with end-over-end rotation before dilution with four volumes of ice-cold RPMI (Invitrogen, Carlsbad, CA). The extracellular medium was collected $(1020 \times g, 5 \mathrm{~min})$ and frozen at $-20^{\circ} \mathrm{C}$ for subsequent analysis.

Study of cytokine and acute phase reactant production in vivo. Neonatal serum was derived from blood samples $(n=33)$ collected into sterile tubes without additives (Greiner BioOne Vacuette, Monroe, NC) at A. Gemelli Hospital (Rome, Italy) following informed consent in accord with an institutionally approved protocol. Cord blood samples $(n=14)$ were collected from healthy newborns immediately after cesarean section (mean GA, 38 wk). Newborns receiving perinatal antibiotics were excluded. Peripheral blood samples were collected from two groups of newborns at d 1-7 (median, $\mathrm{d}$ 2): $1)$ healthy newborns $(n=5)$ born to healthy mothers and collected on d 1-3, and 2) exposed newborns $(n=10)$ born to infected mothers [cytomegalovirus, $n=3$; toxoplasmosis, $n=3$; and $n=1$ each for syphilis, varicellazoster virus (VZV), hepatitis B, and co-infection with hepatitis B and syphilis] but without evidence of neonatal infection by CMV culture and/or conventional serologic assays for pathogen-specific antibodies. Mean GA (38 wk; range, 36-40 wk) and birth weight (3119 g; range, 2040-3970 g) and did not significantly vary between the two groups. For comparison, serum was also prepared from blood samples of 16 healthy adults in Boston (mean age, $24 \mathrm{y}$ ).

Assays for cytokines and acute phase reactants. ELISA for TNF- $\alpha$ (limit of detection $15.6 \mathrm{pg} / \mathrm{mL}$; R \& D Systems, Minneapolis, MN), IL-6 (ultrasensitive assay, limit of detection $0.16 \mathrm{pg} / \mathrm{mL}$; BioSource International, Camarillo, CA), CRP (limit of detection $0.005 \mathrm{mg} / \mathrm{L}$; Life Diagnostics, West Chester, PA), and LBP (limit of detection $1 \mathrm{ng} / \mathrm{mL}$; HyCult Biotechnology, Uden, The Netherlands) were used according to the manufacturer's instructions. All tubes were labeled numerically and the results kept anonymous.

Statistical analysis. Statistical comparisons between groups were made with the Mann-Whitney-Wilcoxon test, or when more than two groups were compared, the Kruskal-Wallis test. Reported correlations are nonparametric Spearman correlations. All $p$ values are two-sided and were considered significant when $p<0.05$.

\section{RESULTS}

We compared TLR-induced production of IL- 6 and TNF- $\alpha$ in whole blood tested in vitro (Fig. 1). To facilitate comparison of neonatal and adult TLR responses, we plotted LPSinduced IL-6 as a function of LPS-induced TNF- $\alpha$ (Fig. 1A). As we have previously described (11), LPS-induced TNF- $\alpha$ production was much greater in adult than in neonatal blood. In marked contrast, however, LPS-induced IL-6 production was at least as great in neonatal as in adult blood (Fig. 1A). When such data were expressed as a ratio of IL-6/TNF- $\alpha$ (Fig. $1 B$ ), neonatal blood was found to produce a $1-2 \log$ greater ratio of IL-6/TNF- $\alpha$ in response to a panel of TLR agonists including poly dI:dC (TLR3), LPS (TLR4), flagellin (TLR5), and CpG DNA (TLR9).

LPS (TLR4) was selected for further analysis. Kinetic analysis at $1,3,5,8$, and $24 \mathrm{~h}$ indicated that whereas LPS

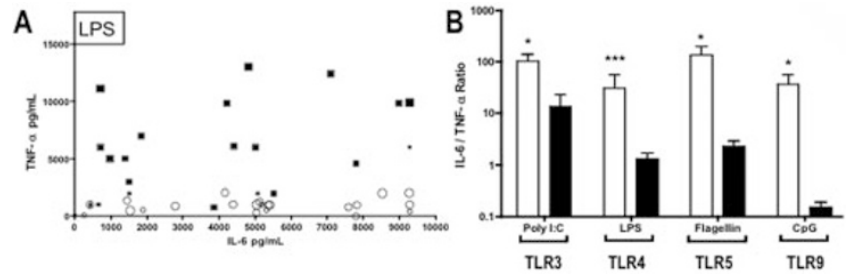

Figure 1. High ratio of IL-6/TNF- $\alpha$ production from neonatal cells in vitro. (A) TNF- $\alpha$ production as a function of that of IL-6 for newborn and adult blood samples. Cytokine production in response to $0.1,1$, and $10 \mathrm{ng} / \mathrm{mL}$ LPS are indicated by small, medium, and large symbols, respectively, with circles representing newborn values and squares representing adult values $(n=$ 5-12). (B) Average IL-6/TNF- $\alpha$ ratios for newborn or adult blood stimulated with the indicated TLR agonists at the concentrations described in "Methods" $(n=3-12)$. IL-6/TNF- $\alpha$ ratios for a given TLR agonist were calculated for each concentration of the agonist tested and then averaged for each study subject, i.e. newborns $(\square)$ or adults (ם). Plotted values represent means + SD. $* p<0.05, * * * p<0.001$.

(TLR4)-induced neonatal TNF- $\alpha$ production was substantially lower than that of adults (Fig. 2A), production of IL-6 was actually greater in neonates across all the time points studied (Fig. 2B).

As we and others have shown by flow cytometry, monocytes are the cells in whole blood predominantly responsible for TLR-induced production of TNF- $\alpha$ (11) and IL-6 [(9) and our results; not shown]. To determine whether such a pattern of cytokine production was evident with a pure cell population, purified neonatal and adult monocytes were cultured in fresh autologous serum. Similar to the results in whole blood, LPS-induced TNF- $\alpha$ was lower in neonatal than adult monocytes but LPS-induced neonatal monocyte IL-6 production was substantially greater than that of adults (Fig. 3A), indicating that the neonatal pattern of cytokine production is not dependent on the presence of other cell types.

We have previously shown that impaired TLR-induced neonatal TNF- $\alpha$ production is in part due to differences between soluble factors in neonatal and adult blood (11). To gain insight into the factors contributing to LPS-induced IL-6 production in neonates, we cultured adult or neonatal monocytes under serum-free conditions (i.e. culture medium), or in autologous or heterologous serum (Fig. 3B). In the absence of serum, neonatal monocytes produced modest amounts of IL-6 that significantly exceeded LPS-induced IL-6 from their adult
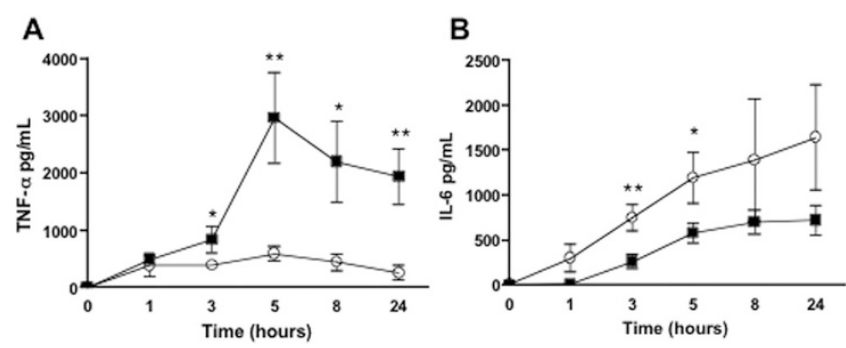

Figure 2. Kinetics of LPS-induced TNF- $\alpha$ and IL-6 production in neonates and adults. Neonatal and adult blood were incubated with $1 \mathrm{ng} / \mathrm{mL}$ LPS for the indicated amount of time before collection of the extracellular medium for TNF- $\alpha$ and IL-6 ELISA. Plotted values are indicated by squares (adults) or circles (newborns) and represent means $\pm \operatorname{SD}(n=3-9 ; * p<0.01$, ** $p<$ $0.001)$. 
A

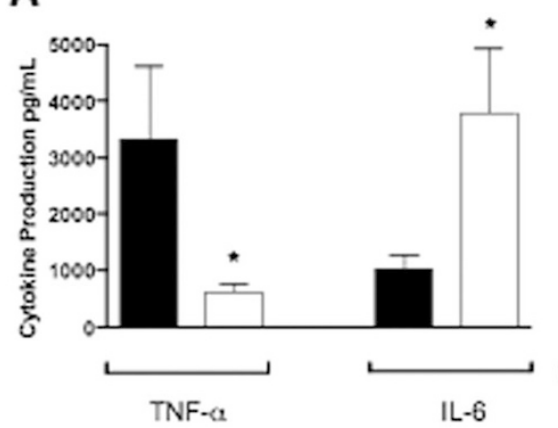

B

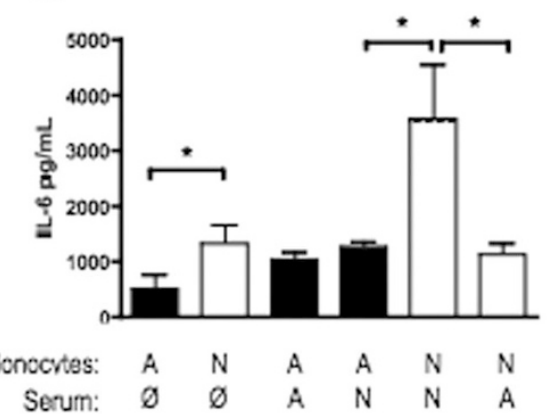

C

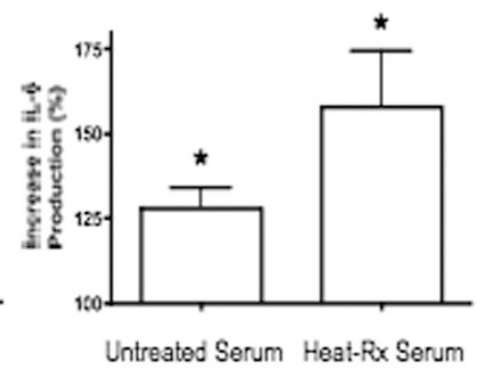

Figure 3. Neonatal monocytes cultured in autologous serum produce a high ratio of IL-6/TNF- $\alpha$. Neonatal and adult monocytes were purified from PBMC by positive selection as described in "Methods." $(A)$ Adult monocytes $(\mathbf{\square})$ or neonatal monocytes $(\square)$ were cultured in fresh autologous serum $\left(10^{7}\right.$ cells/mL) and stimulated with LPS $(1 \mathrm{ng} / \mathrm{mL})$ for $5 \mathrm{~h}$ at $37^{\circ} \mathrm{C}$ before collection of the extracellular medium. Cytokine production was measured by ELISA as described in "Methods." $(B)$ To define the relative contribution of cellular and humoral factors to high LPS-induced TNF- $\alpha$ production, adult $(A)$ or newborn $(N)$ monocytes were cultured in the absence of serum (i.e. in minimal essential medium, $\theta)$, or in fresh adult $(A)$ or newborn $(N)$ serum before stimulation with LPS $(1 \mathrm{ng} / \mathrm{mL})$. (C) To determine whether neonatal serum factor(s) are heat stabile, neonatal, or adult sera were incubated at $56^{\circ} \mathrm{C}$ (or, for comparison, at $4{ }^{\circ} \mathrm{C}$ ) for 30 min before addition to purified neonatal monocytes (final concentration $\left.10^{7} / \mathrm{mL}\right)$ and stimulation with LPS $\left(1 \mathrm{ng} / \mathrm{mL}\right.$ at $37^{\circ} \mathrm{C}$ for $5 \mathrm{~h}$ ). The increase in IL-6 production in the presence of untreated or heat-treated neonatal serum is expressed as a percentage of IL-6 production in the presence of untreated or heat-treated adult serum. Enhancement of LPS-induced IL-6 production by neonatal serum was significant for both conditions and was at least as great in the presence of heat-treated as with untreated serum. Plotted values represent means \pm SD. $n=3-5 ; * p<0.05$.

counterparts. However, robust LPS-induced IL-6 production was only noted when neonatal monocytes were cultured in neonatal serum indicating that both cellular (monocyte) and humoral (serum) factors distinctive to neonates are essential for enhanced LPS-induced IL-6 production. Experiments employing serum that had been heat-treated $\left(56^{\circ} \mathrm{C}, 30 \mathrm{~min}\right)$ revealed that enhancement of LPS-induced IL-6 production was at least as great in the presence of heat-treated neonatal serum as with untreated serum (Fig. $3 C$ ), indicating that the neonatal serum factor(s) that enhance neonatal IL- 6 production are heat stable.

To determine whether a similar neonatal pattern of IL-6/ TNF- $\alpha$ production is evident in vivo, we obtained neonatal cord blood at birth (d 0) and neonatal peripheral blood in the first week of life (d 1-7) from healthy and infection-exposed newborns and also from healthy adults. Concentrations of TNF- $\alpha$ and IL- 6 were quite similar in healthy newborns with healthy mothers and healthy seronegative newborns with in- fected mothers (Fig. 4). These two groups (collected at d 1-7) were therefore pooled in subsequent analyses and compared with cord blood (d 0) and with adult blood. TNF- $\alpha$ concentrations in serum from neonatal peripheral blood obtained at $\mathrm{d}$ 1-7 after birth were similar to concentrations in cord blood, and were significantly lower than in adults (Fig. 4A). In contrast, neonatal serum concentrations of IL-6 at d 1-7 were significantly higher than in cord blood (d 0$)$ and higher than in adults (Fig. 4B). Overall, IL-6/TNF- $\alpha$ ratios at d 1-7 were significantly higher than those in cord blood ( $\mathrm{d} 0$ ) and in adults (Fig. 4C).

To determine whether concentrations of acute phase reactants that are induced by IL- 6 also rise after birth, we measured CRP and LBP in neonatal serum. Concentrations of both CRP (Fig. 5A) and LBP (Fig. 5B) were significantly higher after birth than in cord blood, positively correlating with one another and with IL-6 (pair-wise correlations ranging from 0.50 to 0.62 ).
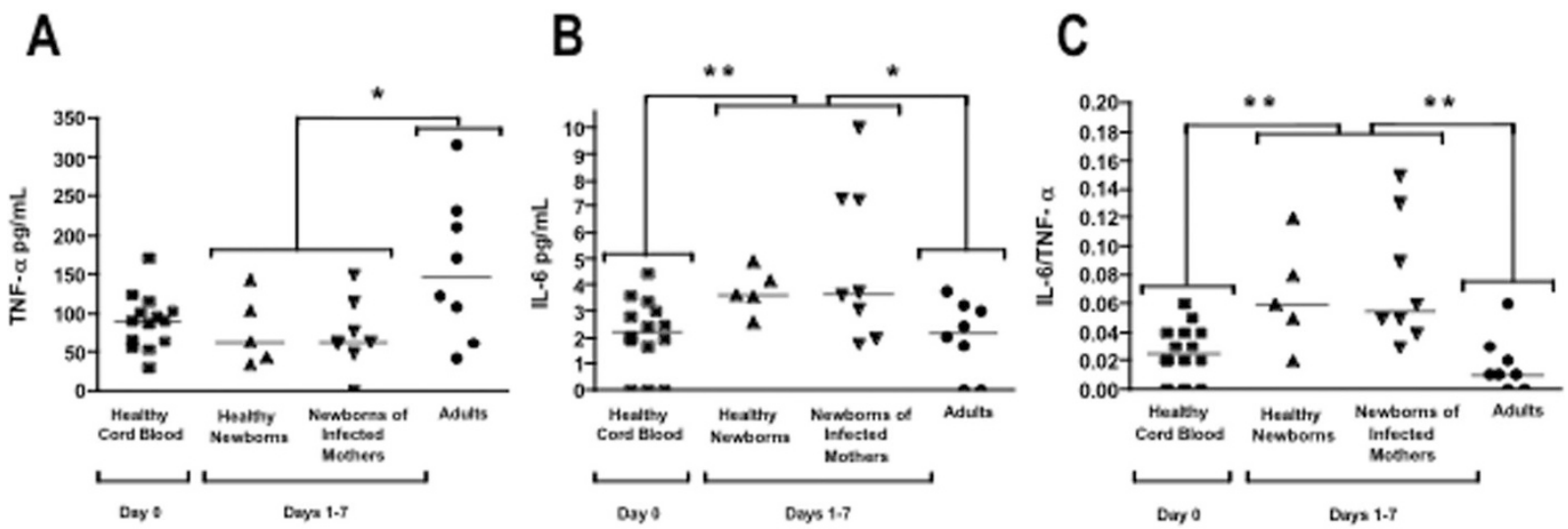

Figure 4. Stable TNF- $\alpha$ but increased IL-6 concentrations in newborns after birth. Serum was prepared from cord blood (d 0) and from peripheral blood (d 1-7), of healthy newborns and newborns born to infected mothers as well as from healthy adult controls. TNF- $\alpha(A)$, IL-6 (B), and IL-6/TNF- $\alpha$ ratios $(C)$ are shown. Horizontal bars indicate median values. $* p<0.05, * * p<0.01$. 
A
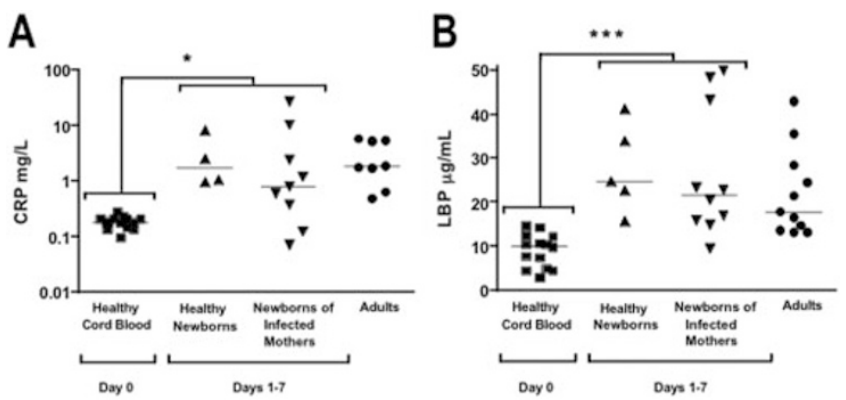

Figure 5. Elevated concentrations of the IL-6-inducible proteins CRP $(A)$ and LBP $(B)$ in neonates. Serum was prepared from cord blood and from peripheral blood, collected at $\mathrm{d} 1-7$, of healthy newborns, newborns born to infected mothers, and healthy adult controls. Horizontal bars indicate median values. $* p<0.05, * * * p<0.001$

\section{DISCUSSION}

Neonatal susceptibility to infection has traditionally been ascribed to "immaturity" of the neonatal immune system. Over the past decade, a more nuanced view has emerged based on evidence of $\mathrm{Th} 2$ polarization of the adaptive neonatal immune response (2). Focusing on innate immunity, our work reveals that with few exceptions (15), there is a strong Th2 bias of TLR-mediated neonatal monocyte cytokine responses under physiologically relevant assay conditions in vitro. Importantly, our study also demonstrates that high IL-6 production and high IL-6/TNF- $\alpha$ ratios are evident during the first few days of life among healthy and infection-exposed newborns in vivo, correlating with elevations in CRP and LBP levels. In aggregate, differences in these markers are highly significant when comparing serum concentrations of newborns at d 1-7 of life with those in newborn cord blood (d 0 ), indicating substantial increases in production of IL-6 (and IL-6 inducible proteins) during the first days of life.

Although we do not know the signals that trigger IL-6 production in the first days after birth in uninfected newborns, it is possible that the initial exposure of the newborn at birth to environmental TLR agonists, including colonizing bacteria (16), might play a role. We therefore speculate that the low basal IL- 6 concentrations in cord sera are due to the fact that there has not yet been substantial exposure to environmental stimuli at the time of cord collection, with a subsequent rise in IL-6 and IL-6-inducible proteins (CRP and LBP) at d 1-7 as the newborn innate immune system responds to a variety of new agonists. Regardless of the identity of the inciting stimulus, the magnitude, consistency, and reproducibility of the neonatal polarization in favor of IL-6 production and against TNF- $\alpha$ both in vitro and in vivo are remarkable and might reflect distinct transcriptional regulation of IL-6 production (17). Of note, CMV (18), herpes simplex virus (HSV) (19), and Toxoplasma (20) express TLR agonists, and HSV induces robust neonatal IL-6 production (19), suggesting that the distinct polarization of neonatal TLR-mediated cytokine production may also be relevant to the pathogenesis of neonatal infections.

In conclusion, neonatal cellular and serum factors program neonatal monocytes to express a high ratio of IL-6/TNF- $\alpha$ in response to TLR agonists in vitro and such a pattern of cytokine production is evident in vivo during the first days after birth. Efforts are underway to further define the physicochemical properties of the heat-stabile IL- 6 enhancing factor of neonatal serum to facilitate its purification. Polarization in favor of IL-6, a cytokine with antiinflammatory and Th2polarizing properties (7), may protect the fetus in utero from potentially harmful Th1 responses that can trigger spontaneous abortion (21) as well as premature delivery and its consequences (22). In addition, IL-6 is an important inducer of the acute phase response and may thereby play an important protective role. However, given the importance of Th1polarizing cytokines in protection against multiple pathogens, IL-6 may also contribute to a Th2-bias that can render the newborn more susceptible to microbial infection. As IL-6 inhibits migration of neutrophils to inflammatory sites (6), we speculate that robust neonatal IL-6 production may also explain the known propensity of newborns to leukopenia in the face of overwhelming sepsis (23). We have recently reported that the distinct function of the neonatal adenosine system accounts for impaired TLR2-mediated monocyte TNF- $\alpha$ production at birth (24). Future studies aimed at further defining the mechanistic basis of neonatal monocyte polarization and exploring the potential clinical utility of pharmacologic modulation of neonatal polarization are indicated.

Acknowledgments. The authors thank Drs. Steven Mizel, Robert Munford, Orazio Ranno, Costantino Romagnoli, and Professor Giuseppe Tortorolo for their support and intellectual input.

\section{REFERENCES}

1. Klein JO, Remington JS 2001 Current concepts of infections of the fetus and newborn infant. In: Remington JS, Klein JO (eds) Infectious Diseases of the Fetus and Newborn Infant. 5th Ed. W.B. Saunders, Philadelphia, pp 1-23

2. Adkins B, Leclerc C, Marshall-Clarke S 2004 Neonatal adaptive immunity comes of age. Nat Rev Immunol 4:553-564

3. Peters AM, Bertram P, Gahr M, Speer CP 1993 Reduced secretion of interleukin-1 and tumor necrosis factor-alpha by neonatal monocytes. Biol Neonate 63:157-162

4. De Wit D, Tonon S, Olislagers V, Goriely S, Boutriaux M, Goldman M, Willems F 2003 Impaired responses to toll-like receptor 4 and toll-like receptor 3 ligands in human cord blood. J Autoimmun 21:277-281

5. Levy O 2005 Innate immunity of the human newborn: distinct cytokine responses to LPS and other Toll-like receptor agonists. J Endotoxin Res 11:113-116

6. Xing Z, Gauldie J, Cox G, Baumann H, Jordana M, Lei XF, Achong MK 1998 IL-6 is an antiinflammatory cytokine required for controlling local or systemic acute inflammatory responses. J Clin Invest 101:311-320

7. Jones SA 2005 Directing transition from innate to acquired immunity: defining a role for IL-6. J Immunol 175:3463-3468

8. Marchini G, Berggren V, Djilali-Merzoug R, Hansson LO 2000 The birth process initiates an acute phase reaction in the fetus-newborn infant. Acta Paediatr 89:10821086

9. Schultz C, Rott C, Temming P, Schlenke P, Moller JC, Bucsky P 2002 Enhanced interleukin-6 and interleukin-8 synthesis in term and preterm infants. Pediatr Res $51: 317-322$

10. Akira S, Takeda K 2004 Toll-like receptor signaling. Nat Rev Immunol 4:499-511

11. Levy O, Zarember KA, Roy RM, Cywes C, Godowski PJ, Wessels MR 2004 Selective impairment of Toll-like receptor-mediated innate immunity in human newborns: neonatal blood plasma reduces monocyte TNF-alpha induction by bacterial lipopeptides, lipopolysaccharide, and imiquimod but preserves response to R-848. J Immunol 173:4627-4634

12. Ulevitch RJ, Tobias PS 1999 Recognition of Gram-negative bacteria and endotoxin by the innate immune system. Curr Opin Immunol 11:19-22

13. Diehl S, Rincon M 2002 The two faces of IL-6 on Th1/Th2 differentiation. Mol Immunol 39:531-536

14. Mizel SB, Honko AN, Moors MA, Smith PS, West AP 2003 Induction of macrophage nitric oxide production by Gram-negative flagellin involves signaling via heteromeric Toll-like receptor 5/Toll-like receptor 4 complexes. J Immunol 170:6217-6223 
15. Levy O, Suter EE, Miller RL, Wessels MR 2006 Unique efficacy of Toll-like receptor agonists in activating human neonatal antigen-presenting cells. Blood, in press

16. Marchini G, Nelson A, Edner J, Lonne-Rahm S, Stavreus-Evers A, Hultenby K 2005 Erythema toxicum neonatorum is an innate immune response to commensal microbes penetrated into the skin of the newborn infant. Pediatr Res 58:613-616

17. Yamamoto M, Yamazaki S, Uematsu S, Sato S, Hemmi H, Hoshino K, Kaisho T, Kuwata $\mathrm{H}$, Takeuchi $\mathrm{O}$, Takeshige $\mathrm{K}$, Saitoh $\mathrm{T}$, Yamaoka S, Yamamoto N, Yamamoto S, Muta T, Takeda K, Akira S 2004 Regulation of Toll/IL-1-receptormediated gene expression by the inducible nuclear protein IkappaBzeta. Nature 430:218-222

18. Compton T 2004 Receptors and immune sensors: the complex entry path of human cytomegalovirus. Trends Cell Biol 14:5-8

19. Kurt-Jones EA, Belko J, Yu C, Newburger PE, Wang J, Chan M, Knipe DM, Finberg RW 2005 The role of toll-like receptors in herpes simplex infection in neonates. J Infect Dis 191:746-748
20. Yarovinsky F, Zhang D, Andersen JF, Bannenberg GL, Serhan CN, Hayden MS, Hieny S, Sutterwala FS, Flavell RA, Ghosh S, Sher A, 2005 TLR11 activation of dendritic cells by a protozoan profilin-like protein. Science 308:1626-1629

21. Makhseed M, Raghupathy R, Azizieh F, Omu A, Al-Shamali E, Ashkanani L 2001 Th1 and Th2 cytokine profiles in recurrent aborters with successful pregnancy and with subsequent abortions. Hum Reprod 16:2219-2226

22. Raghupathy R, Makhseed M, El-Shazly S, Azizieh F, Farhat R, Ashkanani L 2001 Cytokine patterns in maternal blood after premature rupture of membranes. Obstet Gynecol 98:122-126

23. Lewis DB, Wilson CB 2001 Developmental immunology and role of host defenses in neonatal susceptibility to infection. In: Remington JS, Klein JO (eds) Infectious Diseases of the Fetus and Newborn Infant, 5th Ed. W.B. Saunders, Philadelphia, pp $25-138$

24. Levy O, Coughlin M, Cronstein BN, Roy RM, Desai A, Wessels MR 2006 The adenosine system selectively inhibits TLR-mediated TNF- $\alpha$ production in the human newborn. J Immunol, in press 